\title{
Analytical Model of Plug Flow in Microchannels
}

\author{
Zhizhao Che, Teck Neng Wong*, and Nam-Trung Nguyen \\ School of Mechanical and Aerospace Engineering \\ Nanyang Technological University \\ 50 Nanyang Avenue, Singapore, 639798 \\ mtnwong@ntu.edu.sg
}

\begin{abstract}
Plug flow in microchannels is of great importance in microfluidics as it offers many advantages over continuous-flow microfluidics for various applications of lab-on-a-chip platforms. By confining fluids in small plugs of nanoliter scale, recirculation is formed. To increases our understanding of the flow pattern within liquid plugs, an analytical model of liquid plugs moving in microchannels is presented in this paper. The flow field is obtained by solving partial differential equations with corresponding boundary conditions in the microchannel. The comparison with experimental results from micro particle image velocimetry shows reasonable agreement. The model presented in this paper can be used to predict the flow pattern within liquid plugs, and it is helpful for vortex pattern manipulation in various applications of droplet-based microfluidics.
\end{abstract}

Keywords-plug; flow field; analytical model; recirculation; droplet; microfluidics

\section{INTRODUCTION}

With the development of micro-fabrication techniques [1-2], microfluidics gain rapid progress in the recent years [3-5]. As the dimension of the microfluid devices shrinks to micrometer scale, the Reynolds number and the Capillary number are usually very small, and the viscous force and the surface tension force dominate the flow field, while the inertial force and gravitational force are negligible. For plug flow in microchannels, the bubbles/droplets have capsular shapes which completely or partially fill the channel cross-section [68]. With the presence of the interfaces, recirculating flow is formed in the plugs [9]. The recirculating flow field offers many advantages over the continuous-flow counterpart. In liquid plugs, mixing can be significantly enhanced [10], chemical reaction [11] can be achieved in time scale of several millisecond. The acceleration of mixing and reaction are the consequence of the recirculating flow field within the liquid plugs, by which, fluid elements are folded and stretched [1214].

Determining the flow field within liquid plugs is a significant but nontrivial task. Non-intrusive velocity measurement techniques were adopted to measure the flow field at a small space, such as micro particle image velocimetry ( $\mu$ PIV) [15-18]. To measure the flow field of multiphase microfluidics, refractive index of the different phases should be matched to ensure optical path is not distorted by the fluid interface [19].
The numerical methods to simulate the plug flow in microchannel can be divided into two main groups according to the methods to determine the interface shape. The first group assumes a reasonable interface shape to simply the analysis and to reduce the complexity of the problems. Reasonable results can be obtained using this method [20]. In contrast, the second group numerically predicts the interface shape by different interface predicting techniques, such as front tracking [21], moving mesh [22], boundary integration method [23], volume of fluid [24], phase field method [25], level set method [26], lattice-Boltzmann method [27]. These boundary prediction methods are usually complex and computation-time consuming. This is unfavorable especially for applications in which additional processes need to analyze, such as chaos analysis in mixing process [10, 13]. On the contrary, analytical models of plug flow can offer many conveniences because the further analysis can be carried out easily on the known flow fields.

In this paper, we analytically model the plug flow in microchannels. The mathematical equation and the analytical solutions are presented in Section II. The results are provided and analyzed in Section III.

\section{MATHEMATICAL MODELING AND SOLUTIONS}

\section{A. Problem description and assumptions}

Due to the small dimension of the microfluidics devices, the Reynolds number $\operatorname{Re}=\rho V L / \mu$ is usually very small, where $\rho$ is the density of the fluid, $V$ is the characteristic velocity, $L$ is the characteristic length, and $\mu$ is the viscosity of the fluid. Therefore, the inertial force is negligible, while the viscous force dominates the flow in liquid plugs. Fig. 1 shows the schematics of a liquid plug moving in a microchannel. The .liquid plug of incompressible Newtonian fluid is moving at a

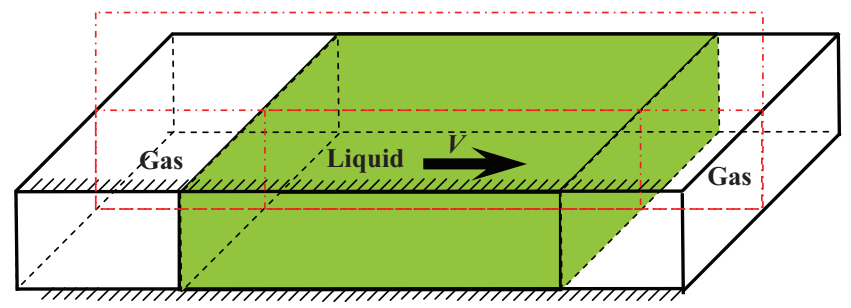

Fig. 1. Schematics of liquid plugs moving (a) in a 2D microchannel, (b) in a capillary with circular cross-section. 
speed $V$ in the microchannel. The surrounding fluid is gas, and its viscosity is negligible comparing with the viscosity of the liquid.

\section{B. Mathematical modelling and analtyical solution}

For plug flow in 2D microchannel, we build a translating Cartesian coordinate on the plug. For the stream function within the liquid plug at Stokes flow condition, the governing equation is [28-29]

$$
\left(\frac{\partial^{4}}{\partial x^{4}}+2 \frac{\partial^{4}}{\partial x^{2} \partial y^{2}}+\frac{\partial^{4}}{\partial y^{4}}\right) \varphi=0
$$

where $\varphi$ is the stream function. The following dimensionless variables are introduced

$$
\begin{gathered}
\hat{x} \equiv x / h \\
\hat{y} \equiv y / h \\
\hat{u}_{x} \equiv u_{x} / V \\
\hat{u}_{y} \equiv u_{y} / V \\
\hat{\varphi} \equiv \varphi /(h V) \\
\hat{L} \equiv L / h
\end{gathered}
$$

where $L$ is the length of the liquid plug, $h$ is the width of the microchannel, and $V$ is the speed of the liquid plugs. Then the governing equation in dimensionless form is

$$
\left(\frac{\partial^{4}}{\partial \hat{x}^{4}}+2 \frac{\partial^{4}}{\partial \hat{x}^{2} \partial \hat{y}^{2}}+\frac{\partial^{4}}{\partial \hat{y}^{4}}\right) \hat{\varphi}=0
$$

By satisfying proper boundary conditions, the dimensionless stream function can be obtained as

$$
\begin{aligned}
\hat{\varphi}(\hat{x}, \hat{y})= & \sum_{n=1,3,5 \cdots}^{\infty} \sin \left(\alpha_{n} \hat{x}\right) \times \\
& {\left[C_{1 n} \cosh \left(\alpha_{n} \hat{y}\right)+C_{2 n} \sinh \left(\alpha_{n} \hat{y}\right)\right.} \\
& \left.+C_{3 n} \hat{y} \cosh \left(\alpha_{n} \hat{y}\right)+C_{4 n} \hat{y} \sinh \left(\alpha_{n} \hat{y}\right)\right]
\end{aligned}
$$

where $\alpha_{n} \equiv n \pi / \hat{L}$. The dimensionless velocity components in the $\hat{x}$ and the $\hat{y}$ directions are respectively

$$
\begin{aligned}
\hat{u}_{x}= & \sum_{n=1,3,5 \cdots}^{\infty} \sin \left(\alpha_{n} \hat{x}\right)\left[C_{1 n} \alpha_{n} \sinh \left(\alpha_{n} \hat{y}\right)+C_{2 n} \alpha_{n} \cosh \left(\alpha_{n} \hat{y}\right)\right. \\
& +C_{3 n} \alpha_{n} \hat{y} \sinh \left(\alpha_{n} \hat{y}\right)+C_{3 n} \cosh \left(\alpha_{n} \hat{y}\right) \\
& \left.+C_{4 n} \alpha_{n} \hat{y} \cosh \left(\alpha_{n} \hat{y}\right)+C_{4 n} \sinh \left(\alpha_{n} \hat{y}\right)\right] \\
\hat{u}_{y}= & -\sum_{n=1,3,5 \cdots}^{\infty} \alpha_{n} \cos \left(\alpha_{n} \hat{x}\right)\left[C_{1 n} \cosh \left(\alpha_{n} \hat{y}\right)+C_{2 n} \sinh \left(\alpha_{n} \hat{y}\right)\right. \\
& \left.+C_{3 n} \hat{y} \cosh \left(\alpha_{n} \hat{y}\right)+C_{4 n} \hat{y} \sinh \left(\alpha_{n} \hat{y}\right)\right]
\end{aligned}
$$

where $C_{1 n}, C_{2 n}, C_{3 n}, C_{4 n}$ are constant coefficients, and they can be determined from the boundary conditions. They are respectively

$$
C_{1 n}=0
$$

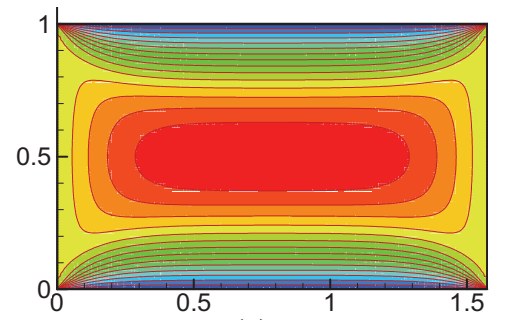

(a)

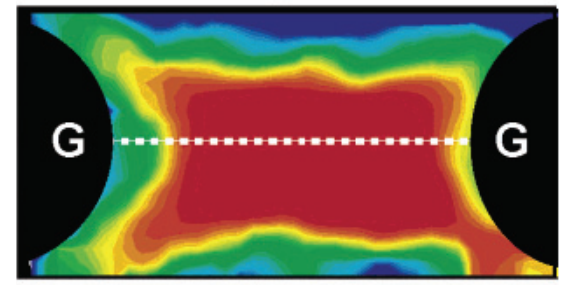

(b)

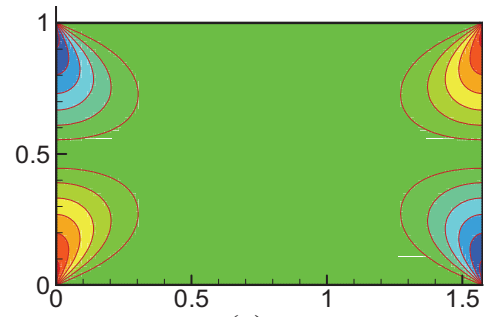

(c)

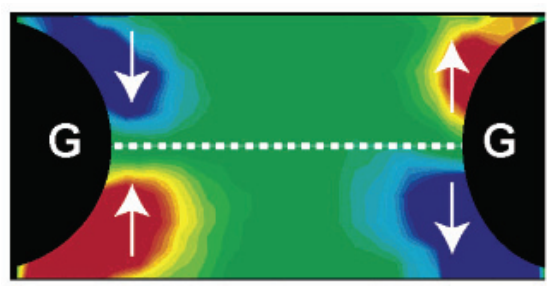

(d)

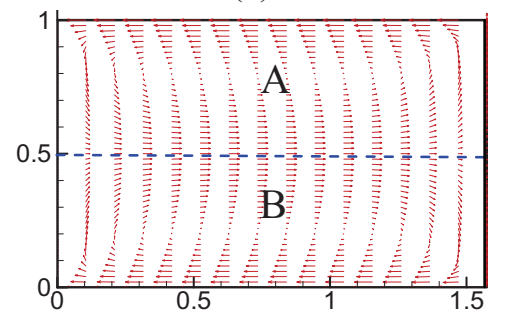

(e)

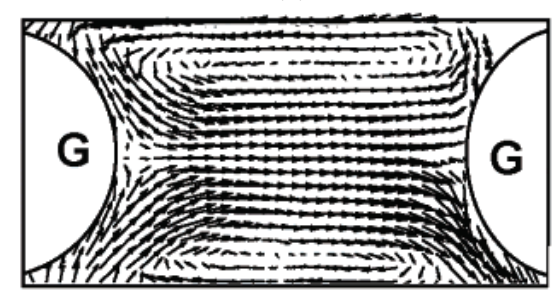

(f)

Fig. 2. Flow field in a plug in a $2 \mathrm{D}$ microchannel. (a) $\hat{u}_{x}$ from the 2D model. (b) $u_{x}$ from $\mu$ PIV [30]. (c) $\hat{u}_{y}$ from the 2D model. (d) $\hat{u}_{y}$ from $\mu$ PIV [30]. (e) Velocity vectors from the analytical model. (f) Velocity vectors from $\mu$ PIV [30]. 


$$
\begin{gathered}
C_{2 n}=-\frac{4}{D_{n} \hat{L} \alpha_{n}} \sinh \left(\alpha_{n}\right)-\frac{4}{D_{n} \hat{L}} \\
C_{3 n}=\frac{4}{D_{n} \hat{L}} \sinh \left(\alpha_{n}\right)+\frac{4 \sinh ^{2}\left(\alpha_{n}\right)}{\hat{L} \alpha_{n} D_{n}} \\
C_{4 n}=\frac{4}{D_{n} \hat{L} \alpha_{n}}\left[-\alpha_{n} \cosh \left(\alpha_{n}\right)+\sinh \left(\alpha_{n}\right)\right. \\
\left.-\sinh \left(\alpha_{n}\right) \cosh \left(\alpha_{n}\right)+\alpha_{n}\right]
\end{gathered}
$$

where

$$
D_{n}=\alpha_{n}^{2}-\sinh ^{2}\left(\alpha_{n}\right)
$$

\section{RESULTS AND DISCUSSION}

\section{A. Validation of the analytical model}

To validate the $2 \mathrm{D}$ model for plug flow in microchannels, we compared our analytical results with velocity field measured from $\mu$ PIV [30], as shown in Fig. 2 . In the $\mu$ PIV measurement (Figs. 2(b, d, f)), a contact angle smaller than 90 degree exists when the liquid plug contacts both the wall of the microchannel. The curvature of the interface is determined by the properties of the wall of the microchannel, the properties of the fluids (both the liquid and the gas), and the flow condition. Though the interface curvature is not considered in the 2D model, the reasonable agreement between those results indicates that the 2D analytical model is capable of predicting the flow fields in the liquid plugs.

\section{B. Flow pattern in the liquid plug}

The vortex pattern in the liquid plug moving in microchannel can be clearly observed in Figs 2(e, f). A pair of symmetric vortices with respect to the center line of the microchannel is shown. The upper vortex $\mathrm{A}$ is circulating in anticlockwise direction, while the lower vortex B in clockwise direction. The two vortices are separated by a streamline (indicated by a dashed line in Fig. 2(e)) and no particle can cross this streamline by advection.

\section{CONCLUSIONS}

In this paper, we analytically investigated the flow field in liquid plugs moving in two dimensional microchannels. The Stokes flow within the liquid plugs is modeled by partial differential equations and the boundary conditions are satisfied. The results are compared with experimental results. The reasonable agreement between the results from the analytical model and the flow field from $\mu$ PIV shows that the models presented in this paper can capture the flow feature in the liquid plugs. These models can be used for various applications of droplet-based microfluidics, such as vortex pattern manipulation in plugs, heat and mass transfer analysis, design of micromixers and microreactors, synthesis and analysis in droplets.

\section{REFERENCES}

[1] J. C. McDonald, D. C. Duffy, J. R. Anderson, D. T. Chiu, H. Wu, et al., "Fabrication of microfluidic systems in poly(dimethylsiloxane)," Electrophoresis., vol. 21, pp. 27-40, 2000.

[2] M. J. Madou, Fundamentals of Microfabrication: The Science of Miniaturization, 2nd ed.: Boca Raton, FL: CRC Press, 2002.

[3] C. M. Ho and Y. C. Tai, "Micro-electro-mechanical-systems (MEMS) and fluid flows," Annu. Rev. Fluid. Mech., vol. 30, pp. 579-612, 1998.

[4] T. M. Squires and S. R. Quake, "Microfluidics: Fluid physics at the nanoliter scale," Rev. Mod. Phys., vol. 77, pp. 977-1026, 2005.

[5] H. A. Stone, A. D. Stroock, and A. Ajdari, "Engineering flows in small devices: Microfluidics toward a lab-on-a-chip," Annu. Rev. Fluid. Mech., vol. 36, pp. 381-411, 2004.

[6] P. Angeli and A. Gavriilidis, "Hydrodynamics of Taylor flow in small channels: A review," P. I. Mech. Eng. C-J. Mec, vol. 222, pp. 737-751, 2008.

[7] A. Günther and K. F. Jensen, "Multiphase microfluidics: From flow characteristics to chemical and materials synthesis," Lab Chip, vol. 6, pp. 1487-1503, 2006.

[8] S. Y. Teh, R. Lin, L. H. Hung, and A. P. Lee, "Droplet microfluidics," Lab Chip, vol. 8, pp. 198-220, 2008.

[9] K. Handique and M. A. Burns, "Mathematical modeling of drop mixing in a slit-type microchannel," J. Micromech. Microeng, vol. 11, pp. 548554, 2001.

[10]H. Song, M. R. Bringer, J. D. Tice, C. J. Gerdts, and R. F. Ismagilov, "Experimental test of scaling of mixing by chaotic advection in droplets moving through microfluidic channels," Appl. Phys. Lett., vol. 83, pp. 4664-4666, 2003.

[11]H. Song, J. D. Tice, and R. F. Ismagilov, "A microfluidic system for controlling reaction networks in time," Angew. Chem. Int. Edit., vol. 42, pp. 768-772, 2003.

[12]H. Aref, "Stirring by chaotic advection," J. Fluid. Mech., vol. 143, pp. 121, 1984.

[13]J. M. Ottino, The kinematics of mixing: stretching, chaos, and transport, 1989.

[14]H. Aref, "The development of chaotic advection," Phys. Fluids., vol. 14, pp. 1315-1325, 2002.

[15]J. G. Santiago, S. T. Wereley, C. D. Meinhart, D. J. Beebe, and R. J. Adrian, "A particle image velocimetry system for microfluidics," Exp. Fluids., vol. 25, pp. 316-319, 1998.

[16]D. Sinton, "Microscale flow visualization," Microfluid. Nanofluid., vol. 1, pp. 2-21, 2004.

[17]R. Lindken, M. Rossi, S. Große, and J. Westerweel, "Micro-Particle Image Velocimetry (PIV): Recent developments, applications, and guidelines," Lab Chip, vol. 9, pp. 2551-2567, 2009.

[18]S. T. Wereley and C. D. Meinhart, "Recent advances in Micro-Particle Image Velocimetry," Annu. Rev. Fluid. Mech., vol. 42, pp. 557-576, 2010.

[19]M. Oishi, H. Kinoshita, T. Fujii, and M. Oshima, "Confocal micro-PIV measurement of droplet formation in a T-shaped micro-junction," J. Phys. Conf. Ser., p. 012061, 2009.

[20]N. Harries, J. R. Burns, D. A. Barrow, and C. Ramshaw, "A numerical model for segmented flow in a microreactor," Int. J. Heat Mass. Tran, vol. 46, pp. 3313-3322, 2003. 
[21]G. Tryggvason, B. Bunner, A. Esmaeeli, D. Juric, N. Al-Rawahi, et al., "A front-tracking method for the computations of multiphase flow," J. Comput. Phys., vol. 169, pp. 708-759, 2001.

[22]B. Perot and R. Nallapati, "A moving unstructured staggered mesh method for the simulation of incompressible free-surface flows," J. Comput. Phys., vol. 184, pp. 192-214, 2003.

[23]T. Y. Hou, J. S. Lowengrub, and M. J. Shelley, "Boundary integral methods for multicomponent fluids and multiphase materials," J. Comput. Phys., vol. 169, pp. 302-362, 2001.

[24]R. Scardovelli and S. Zaleski, "Direct numerical simulation of freesurface and interfacial flow," Annu. Rev. Fluid. Mech., vol. 31, pp. 567$603,1999$.

[25]D. M. Anderson, G. B. McFadden, and A. A. Wheeler, "Diffuse-interface methods in fluid mechanics," Annu. Rev. Fluid. Mech., vol. 30, pp. 139$165,1998$.
[26]S. Osher and R. P. Fedkiw, Level set methods and dynamic implicit surfaces. New York: Springer, 2003.

[27]S. Chen and G. D. Doolen, "Lattice Boltzmann method for fluid flows," Annu. Rev. Fluid. Mech., vol. 30, pp. 329-364, 1998.

[28]P. N. Shankar, Slow Viscous Flows -- Qualitative Features and Quantitative Analysis Using Complex Eigenfunction Expansions: Imperial College Press, 2007.

[29]J. Happel and H. Brenner, Low Reynolds number hydrodynamics: with special applications to particulate media, 2nd ed. The Hague: Martinus Nijhoff Publishers, 1983.

[30]A. Günther, M. Jhunjhunwala, M. Thalmann, M. A. Schmidt, and K. F. Jensen, "Micromixing of miscible liquids in segmented gas-liquid flow," Langmuir., vol. 21, pp. 1547-1555, 2005. 